\title{
COMPARATIVE STUDY OF GLUCOSE AND FRUCTOSE METABO- LISM IN INFANTS WITH REFERENCE TO UTILIZATION AND TO THE ACCUMULATION OF GLYCOLYTIC INTERMEDIATES ${ }^{1}$
}

\author{
By ROBERT KAYE, MARGARET L. WILLIAMS, AND GIULIO BARBERO \\ (From the Children's Hospital of Philadelphia, and the Department of Pediatrics, University of \\ Pennsylvania School of Medicine, Philadelphia, Pa.)
}

(Submitted for publication September 12, 1957; accepted September 26, 1957)

A number of studies of the comparative utilization $^{2}$ of intravenously administered glucose and fructose have been carried out in adults $(1-4)$. The results have almost uniformly favored fructose, but have been concerned for the most part with infusions which were of short duration and quantitatively inadequate from the standpoint of caloric maintenance. The caloric equivalents of the differences in the excretion of the sugars were clinically insignificant.

Comparative utilization studies involving amounts of carbohydrate adequate for the provision of caloric maintenance have been carried out by Strub, Best, Consolazio, and Grossman (4) and by Jonxis and Huisman (5). The former group administered fructose and invert sugar (equal parts of glucose and fructose) in $300 \mathrm{Gm}$. quantities over a period of three hours and observed approximately 95 per cent retention in both cases. Jonxis and Huisman, who carried out the only study previously reported in children, were unable to elicit any difference in utilization of glucose and invert sugar. However, the rate of carbohydrate administration employed in their experiments, which were of 24 hours duration, was sufficiently slow to permit essentially complete utilization of both sugars.:

In conflict with the consensus of a large number of studies, Beal, Smith, and Frost (6) have found fructose losses in the urine to exceed those of glucose. These authors have explained the discrepancy between their results and those of others on the basis of a delayed excretion of fruc-

\footnotetext{
1 This work was supported by grants from the National Institutes of Health and the Mead Johnson Company.

2 The term "utilized" is used to denote the difference between carbohydrate input and renal excretion during the duration of the study period.
}

tose following completion of the infusion and return of the blood level to normal.

The importance of parenteral alimentation in infants suggested that a comparative study of the utilization of glucose and fructose in this age group would be of value. The present study was designed to carry out the comparison at a level of intake which would provide sufficient calories for caloric maintenance.

The rates of administration were selected $a$ ) to determine whether fructose utilization equals the maximum generally considered to obtain for glucose ( $1 \mathrm{Gm}$. per $\mathrm{Kg}$. per hour) (7) and $b$ ) to compare utilization of the two sugars when administered at rates in excess of the infants' capacity for relatively complete glucose utilization $(2 \mathrm{Gm}$. per Kg. per hour).

The appearance of certain disadvantageous side effects during the course of fructose infusions at the more rapid rate, and several reports from other laboratories of the development of acidosis with fructose administration $(8,9)$ lead to investigation of the attendant acid-base changes. A further study was carried out to compare the extent of accumulation of certain acid metabolites and the magnitude of the acid-base disturbance which results from the rapid infusion of glucose and fructose. The data derived from this study have bearing on the clinical use of these two carbohydrates.

\section{MATERIALS AND METHODS}

Studies on utilization. Eleven male infants convalescent from neurosurgical procedures were used as subjects. Their average age was 6.4 months (range, 2 to 16 months) and their weight averaged $7.2 \mathrm{Kg}$., (range, 5.4 to $10 \mathrm{Kg}$.). They were divided into two groups. Group A consisted of three individuals given infusions of each of the carbohydrates at a rate of approximately $1.0 \mathrm{Gm}$. per Kg. per hour. Group B consisted of eight subjects who received a total of 17 hexose infusions at a rate 
TABLE I

Composition of solutions

\begin{tabular}{|c|c|c|c|c|c|c|c|}
\hline \multirow[b]{2}{*}{$\begin{array}{l}\text { Hexose } \\
\mathrm{Na} \\
\mathrm{K} \\
\mathrm{Cl} \\
\text { Phosphate } \\
\text { pH }\end{array}$} & \multirow[b]{2}{*}{$\begin{array}{l}\mathrm{Gm} . / 100 \mathrm{ml} . \\
\mathrm{mEq} \cdot / \mathrm{L} . \\
\mathrm{mEq} \cdot / \mathrm{L} . \\
\mathrm{mEq} \cdot / \mathrm{L} . \\
\mathrm{mM} / \mathrm{L} .\end{array}$} & \multicolumn{3}{|c|}{ Fructose } & \multicolumn{3}{|c|}{ Glucose } \\
\hline & & $\begin{array}{c}5.0 \\
33.5 \\
17.0 \\
42.7 \\
5.9 \\
4.30\end{array}$ & $\begin{array}{c}7.2 \\
33.5 \\
16.7 \\
44.4 \\
6.0 \\
4.30\end{array}$ & $\begin{array}{c}10.1 \\
33.0 \\
16.7 \\
44.4 \\
6.0 \\
4.30\end{array}$ & $\begin{array}{c}5.1 \\
33.5 \\
17.4 \\
44.4 \\
5.0 \\
4.35\end{array}$ & $\begin{array}{c}7.2 \\
33.0 \\
17.2 \\
42.7 \\
6.0 \\
4.45\end{array}$ & $\begin{array}{c}10.1 \\
32.5 \\
17.0 \\
42.7 \\
5.9 \\
4.45\end{array}$ \\
\hline
\end{tabular}

of $2.0 \mathrm{Gm}$. per $\mathrm{Kg}$. per hour. With the exception of one subject who received three infusions, one of glucose and two of fructose, all were given one infusion of each of the carbohydrates, with the initial carbohydrate randomized. The glucose and fructose were infused on different days with the subject in equivalently good states of hydration on both days.

The solutions were administered at a rate of approximately $25 \mathrm{ml}$. per $\mathrm{Kg}$. per hour for a period of six hours except for the experiments on Subjects 3 and 10, which were of four hours duration. A positive pressure pump was used to secure a uniform rate of administration. The solutions, whose compositions are given in Table I, were prepared by the Mead Johnson Research Laboratories. They were designed to provide constant amounts of water and electrolytes and increasing hexose concentrations of $5.0,7.15$, and $10.0 \mathrm{Gm}$. per $100 \mathrm{ml}$.

Observations of pulse, respiratory rate, and liver size were made at intervals of two hours.

Analyses of the blood were carried out prior to the infusion for the hematocrit and concentrations of glucose and fructose, sodium and potassium. Carbohydrate analyses were repeated at intervals of two hours during the infusion and one hour after its completion. Final determinations of sodium and potassium and of the hematocrit were performed at the end of the infusion. Glucose and hematocrit determinations were done on capillary blood. The other analyses were done on serum obtained from venous blood. The methods used were the Nelson modification of the Somogyi method for total reducing substance (10), Schreiner's modification of the Roe method for fructose (11), and flame photometry for sodium and potassium.

Complete urine collections were obtained. Separate analyses were carried out on aliquots of the collections at two hourly intervals during the infusion, at one hour postinfusion and finally for an additional four to eight hours. Urine was analyzed for glucose and fructose, sodium, potassium and specific gravity.

The quantity of carbohydrate excreted in the urine following the first postinfusion hour was negligible in all cases and was therefore omitted from the calculations. This finding is at variance with the observations of Beal, Smith, and Frost (6).

Study of acid-base changes. The experimental group here consisted of 15 hospital in-patients who were convalescent from a variety of diseases. They ranged in age from 11 weeks to 2 years and in weight from 5.3 to 12.7
$\mathrm{Kg}$. Seven subjects received intravenous infusions of a 10 per cent fructose solution with electrolytes at a rate of $2 \mathrm{Gm}$. per $\mathrm{Kg}$. per hour and eight subjects received a glucose solution of similar electrolyte concentration at the same rate. The duration of the infusion was six hours except for two fructose studies which were limited to four hours because of obvious evidence of progressive acidosis.

The infused solutions were similar to the 10 per cent hexose solutions of the first experiments, except that they contained somewhat less chloride (approximately $35 \mathrm{mEq}$. per L.). The infusion technique was the same but blood sampling in these experiments was done through an indwelling Cournand needle which was placed in the femoral artery or vein 15 to 30 minutes before the start of the infusion, thus avoiding struggling during the sampling which might have elevated lactate and pyruvate concentrations in the blood. Samples were taken prior to the start, at the fourth hour, and at the end (sixth hour) of the infusion and the following studies were done: whole blood $\mathrm{pH}$ by a Cambridge $\mathrm{pH}$ meter, whole blood carbon dioxide by the technique of Van Slyke and Neill (12), plasma sodium and potassium by flame photometry, plasma chloride by the method of Schales and Schales (13), whole blood lactate by the procedure of Barker and Summerson (14), and whole blood pyruvate by the direct method of Friedemann and Haugen (15) which measures total keto acids rather than pyruvate alone.

A total urine collection was made for the period of the infusion plus the following hour and kept at a $\mathrm{pH}$ of approximately 1.0 in ice. The urine was analyzed for sodium, potassium, lactate, pyruvate and total reducing substance by the methods used above and for fructose by the Roe method as modified by Higashi and Peters (16).

\section{RESULTS}

\section{Studies on utilization}

The rate of infusion of the subjects in Group A (Table II) averaged $1.07 \mathrm{Gm}$. per $\mathrm{Kg}$. per hour (range, 0.89 to 1.22 ) and 1.06 (range, 0.81 to 1.30) for glucose and fructose, respectively. In these experiments, the retention of both sugars was essentially complete and equal as indicated by the fact that only 1.6 per cent and 3.6 per cent of 
TABLE II

Balance data during hexose infusions-Group $A$

\begin{tabular}{|c|c|c|c|c|c|c|c|c|c|c|}
\hline \multirow{3}{*}{$\begin{array}{c}\text { Subject } \\
\text { Subj. } 1(\mathrm{Fe}) \\
\text { age, } 7 \mathrm{mo} \\
\text { wt., } 7.7 \mathrm{Kg} .\end{array}$} & \multirow{2}{*}{$\begin{array}{l}\text { Infusion } \\
\text { time } \\
\text { hrs. }\end{array}$} & & \multicolumn{2}{|c|}{$\begin{array}{c}\text { Water } \\
m l .\end{array}$} & \multirow{2}{*}{\multicolumn{2}{|c|}{$\frac{\begin{array}{c}\text { Hexose } \\
\text { Gm. }\end{array}}{\text { Glucose Fructose }}$}} & \multirow{2}{*}{\multicolumn{2}{|c|}{$\frac{\underset{m E q}{\mathrm{Na}}}{\text { Glucose Fructose }}$}} & \multirow{2}{*}{\multicolumn{2}{|c|}{$\frac{\underset{m E q}{\mathbf{K}}}{\text { Glucose Fructose }}$}} \\
\hline & & & Glucose & Fructose & & & & & & \\
\hline & 6 & $\begin{array}{l}\text { Intake } \\
\quad \text { perKg./hr. } \\
\text { total } \\
\text { Output } \\
\text { Retention } \\
\text { \% Excreted }\end{array}$ & $\begin{array}{l}21.4 \\
990 \\
826 \\
164 \\
83.4\end{array}$ & $\begin{array}{c}16.0 \\
740 \\
400 \\
340 \\
54.0\end{array}$ & $\begin{array}{c}1.09 \\
50.4 \\
0.3 \\
50.1 \\
0.6\end{array}$ & $\begin{array}{c}0.81 \\
37.3 \\
0.9 \\
36.4 \\
2.4\end{array}$ & $\begin{array}{l}0.72 \\
33.2 \\
22.0 \\
11.2 \\
66.3\end{array}$ & $\begin{array}{c}0.54 \\
24.8 \\
2.7 \\
22.1 \\
10.9\end{array}$ & $\begin{array}{c}0.37 \\
17.2 \\
12.2 \\
5.0 \\
70.9\end{array}$ & $\begin{array}{l}0.27 \\
12.6 \\
10.4 \\
2.2 \\
82.5\end{array}$ \\
\hline $\begin{array}{l}\text { Subj. } 2(\mathrm{Fu}) \\
\text { age, } 7 \mathrm{mo} \text {. } \\
\text { wt., } 8.8 \mathrm{Kg} .\end{array}$ & 6 & $\begin{array}{l}\text { Intake } \\
\quad \text { per } \mathrm{Kg} . / \mathrm{hr} . \\
\text { total } \\
\text { Output } \\
\text { Retention } \\
\text { \% Excreted }\end{array}$ & $\begin{array}{l}17.5 \\
923 \\
863 \\
60 \\
93.5\end{array}$ & $\begin{array}{c}21.0 \\
1,111 \\
730 \\
381 \\
65.7\end{array}$ & $\begin{array}{c}0.89 \\
47.0 \\
1.5 \\
45.5 \\
3.2\end{array}$ & $\begin{array}{c}1.06 \\
56.0 \\
2.4 \\
53.6 \\
4.3\end{array}$ & $\begin{array}{l}0.58 \\
30.9 \\
11.0 \\
19.9 \\
35.6\end{array}$ & $\begin{array}{l}0.70 \\
37.2 \\
22.7 \\
14.5 \\
61.0\end{array}$ & $\begin{array}{c}0.30 \\
16.1 \\
7.1 \\
9.0 \\
44.1\end{array}$ & $\begin{array}{c}0.36 \\
18.9 \\
7.7 \\
11.2 \\
40.7\end{array}$ \\
\hline $\begin{array}{c}\text { Subj. } 3(\mathrm{Re}) \\
\text { age, } 4 \mathrm{mo} \\
\text { wt., } 7.2 \mathrm{Kg} .\end{array}$ & 4 & $\begin{array}{l}\text { Intake } \\
\quad \text { per Kg./hr. } \\
\text { total } \\
\text { Output } \\
\text { Retention } \\
\text { \% Excreted }\end{array}$ & $\begin{array}{l}23.9 \\
690 \\
515 \\
175 \\
74.6\end{array}$ & $\begin{array}{l}25.8 \\
743 \\
590 \\
153 \\
79.4\end{array}$ & $\begin{array}{c}1.22 \\
35.1 \\
0.4 \\
34.7 \\
1.1\end{array}$ & $\begin{array}{c}1.30 \\
37.4 \\
1.5 \\
35.9 \\
4.0\end{array}$ & $\begin{array}{l}0.80 \\
23.1 \\
2.4 \\
20.7 \\
10.4\end{array}$ & $\begin{array}{l}0.86 \\
24.9 \\
11.8 \\
13.1 \\
47.4\end{array}$ & $\begin{array}{c}0.42 \\
12.0 \\
4.4 \\
7.6 \\
36.7\end{array}$ & $\begin{array}{c}0.44 \\
12.6 \\
6.3 \\
6.3 \\
50.0\end{array}$ \\
\hline Average & & $\begin{array}{l}\text { Intake } \\
\text { per Kg. } / \mathrm{hr} . \\
\% \text { Excreted }\end{array}$ & $\begin{array}{l}20.9 \\
83.8\end{array}$ & $\begin{array}{l}20.9 \\
66.4\end{array}$ & $\begin{array}{l}1.07 \\
1.6\end{array}$ & $\begin{array}{l}1.06 \\
3.6\end{array}$ & $\begin{array}{c}0.70 \\
37.4\end{array}$ & $\begin{array}{c}0.70 \\
39.8\end{array}$ & $\begin{array}{c}0.36 \\
50.6\end{array}$ & $\begin{array}{c}0.36 \\
57.7\end{array}$ \\
\hline
\end{tabular}

the infused carbohydrates were excreted in the glucose and fructose experiments, respectively. No noteworthy differences were seen in the excretions of water, sodium and potassium in relation to the sugar infused.

The infusions administered to the eight subjects in Group B (Table III) were carried out at a rate known to exceed the infants' capacity for essentially complete utilization of glucose. Favor- able circumstances were created thereby for the possible demonstration of a greater capacity for fructose utilization. The average percentage of the hexose intake excreted in the urine in the glucose experiments, 20.0 per cent, was significantly greater than that of the fructose experiments, 9.9 per cent $(p<0.02)$. The average sodium excretion associated with glucose infusion was 79 per cent of the intake, which is significantly

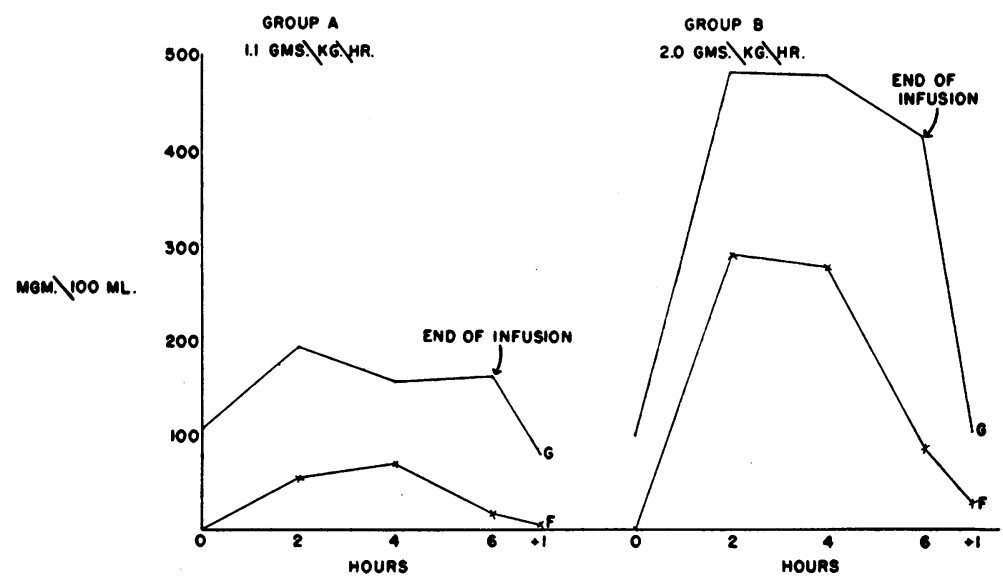

Fig. 1. Average Blood Levels During and One Hour After Infusion

The dots represent blood levels of total reducing substance during and after glucose infusion. The crosses are blood levels with fructose infusion. 
TABLE III

Balance data during hexose infusions-Group $B$

\begin{tabular}{|c|c|c|c|c|c|c|c|c|c|c|}
\hline \multirow{3}{*}{$\begin{array}{c}\text { Subject } \\
\text { Subj. } 4(\mathrm{Ha}) \\
\text { age, } 6 \mathrm{mo} . \\
\text { wt., } 8.5 \mathrm{Kg} .\end{array}$} & \multirow{3}{*}{$\begin{array}{l}\text { Infusion } \\
\text { time } \\
\text { hrs. }\end{array}$} & & \multicolumn{2}{|c|}{$\begin{array}{c}\text { Water } \\
m l .\end{array}$} & \multirow{2}{*}{\multicolumn{2}{|c|}{$\frac{\begin{array}{c}\text { Hexose } \\
\mathrm{Gm} .\end{array}}{\text { Glucose Fructose }}$}} & \multicolumn{2}{|c|}{$\underset{m E q .}{\mathrm{Na}}$} & \multirow{2}{*}{\multicolumn{2}{|c|}{$\frac{\underset{m E q .}{\mathrm{K}}}{\text { Glucose Fructose }}$}} \\
\hline & & & Glucose & Fructose & & & Glucose & Fructose & & \\
\hline & & $\begin{array}{l}\text { Intake } \\
\quad \text { per } \mathrm{Kg} . / \mathrm{hr} . \\
\text { total } \\
\text { Output } \\
\text { Retention } \\
\text { \% Excreted }\end{array}$ & $\begin{array}{c}21.8 \\
1,111 \\
841 \\
270 \\
75.7\end{array}$ & $\begin{array}{c}20.1 \\
1,024 \\
776 \\
248 \\
75.8\end{array}$ & $\begin{array}{l}1.57 \\
80.2 \\
15.6 \\
64.6 \\
19.4\end{array}$ & $\begin{array}{c}1.44 \\
73.6 \\
5.9 \\
67.7 \\
8.0\end{array}$ & $\begin{array}{l}0.72 \\
36.7 \\
29.5 \\
7.2 \\
80.4\end{array}$ & $\begin{array}{c}0.67 \\
34.3 \\
24.8 \\
9.5 \\
72.3\end{array}$ & $\begin{array}{c}0.37 \\
19.1 \\
11.9 \\
7.2 \\
62.3\end{array}$ & $\begin{array}{c}0.33 \\
17.1 \\
8.5 \\
8.6 \\
49.7\end{array}$ \\
\hline $\begin{array}{c}\text { Subj. } 5(\mathrm{Ch}) \\
\text { age, } 3 \mathrm{mo} . \\
\text { wt., } 6.5 \mathrm{Kg} .\end{array}$ & 6 & $\begin{array}{l}\text { Intake } \\
\quad \text { per } \mathrm{Kg} . / \mathrm{hr} . \\
\quad \text { total } \\
\text { Output } \\
\text { Retention } \\
\text { \% Excreted }\end{array}$ & $\begin{array}{c}27.5 \\
1,072 \\
930 \\
142 \\
86.7\end{array}$ & $\begin{array}{c}27.2 \\
1,063 \\
929 \\
134 \\
87.4\end{array}$ & $\begin{array}{l}1.98 \\
77.4 \\
12.1 \\
65.3 \\
15.6\end{array}$ & $\begin{array}{c}1.96 \\
76.4 \\
8.6 \\
67.8 \\
11.2\end{array}$ & $\begin{array}{l}0.91 \\
35.4 \\
25.6 \\
9.8 \\
72.3\end{array}$ & $\begin{array}{r}0.91 \\
35.6 \\
50.2 \\
-14.6 \\
141.0\end{array}$ & $\begin{array}{c}0.47 \\
18.4 \\
11.2 \\
7.2 \\
60.9\end{array}$ & $\begin{array}{l}0.45 \\
17.7 \\
11.7 \\
6.0 \\
66.1\end{array}$ \\
\hline $\begin{array}{c}\text { Subj. } 6(\mathrm{Gr}) \\
\text { age, } 9 \mathrm{mo} \text {. } \\
\text { wt., } 5.8 \mathrm{Kg} .\end{array}$ & 6 & $\begin{array}{l}\text { Intake } \\
\quad \text { per } \mathrm{Kg} . / \mathrm{hr} . \\
\text { total } \\
\text { Output } \\
\text { Retention } \\
\text { \% Excreted }\end{array}$ & $\begin{array}{c}30.5 \\
1,062 \\
892 \\
170 \\
84.0\end{array}$ & $\begin{array}{c}30.0 \\
1,045 \\
878 \\
167 \\
84.0\end{array}$ & $\begin{array}{l}2.20 \\
76.7 \\
20.5 \\
56.2 \\
26.7\end{array}$ & $\begin{array}{l}2.16 \\
75.1 \\
10.0 \\
65.1 \\
13.3\end{array}$ & $\begin{array}{l}1.00 \\
35.0 \\
31.5 \\
3.5 \\
90.0\end{array}$ & $\begin{array}{c}1.00 \\
35.0 \\
42.1 \\
-7.1 \\
120.3\end{array}$ & $\begin{array}{c}0.52 \\
18.3 \\
9.3 \\
9.0 \\
50.8\end{array}$ & $\begin{array}{c}0.50 \\
17.4 \\
8.8 \\
8.6 \\
50.6\end{array}$ \\
\hline $\begin{array}{l}\text { Subj. } 7 \text { (Ro) } \\
\text { age, } 5 \mathrm{mo} . \\
\text { wt., } 5.7 \mathrm{Kg} .\end{array}$ & 6 & $\begin{array}{l}\text { Intake } \\
\quad \text { per } \mathrm{Kg} . / \mathrm{hr} \text {. } \\
\quad \text { total } \\
\text { Output } \\
\text { Retention } \\
\text { \% Excreted }\end{array}$ & $\begin{array}{c}21.7 \\
744 \\
498 \\
246 \\
66.9\end{array}$ & $\begin{array}{l}22.3 \\
762 \\
672 \\
90 \\
88.2\end{array}$ & $\begin{array}{r}1.57 \\
53.7 \\
1.9 \\
51.8 \\
3.5\end{array}$ & $\begin{array}{c}1.60 \\
54.8 \\
4.1 \\
50.7 \\
7.5\end{array}$ & $\begin{array}{l}0.72 \\
24.5 \\
11.5 \\
13.0 \\
46.9\end{array}$ & $\begin{array}{c}0.74 \\
25.5 \\
36.1 \\
-10.6 \\
141.6\end{array}$ & $\begin{array}{c}0.37 \\
12.8 \\
4.9 \\
7.9 \\
38.3\end{array}$ & $\begin{array}{c}0.37 \\
12.7 \\
7.6 \\
5.1 \\
59.8\end{array}$ \\
\hline $\begin{array}{c}\text { Subj. } 8(\mathrm{Ba}) \\
\text { age, } 5 \mathrm{mo} . \\
\text { wt., } 6.4 \mathrm{Kg} .\end{array}$ & 6 & $\begin{array}{l}\text { Intake } \\
\text { per } \mathrm{Kg} . / \mathrm{hr} . \\
\text { total } \\
\text { Output } \\
\text { Retention } \\
\text { \% Excreted }\end{array}$ & $\begin{array}{c}27.1 \\
1,042 \\
873 \\
169 \\
83.8\end{array}$ & $\begin{array}{l}25.7 \\
989 \\
825 \\
164 \\
83.4\end{array}$ & $\begin{array}{l}1.96 \\
75.2 \\
17.4 \\
57.8 \\
23.1\end{array}$ & $\begin{array}{c}1.85 \\
71.1 \\
5.5 \\
65.6 \\
7.7\end{array}$ & $\begin{array}{l}0.89 \\
34.4 \\
29.4 \\
5.0 \\
85.5\end{array}$ & $\begin{array}{c}0.86 \\
33.1 \\
34.1 \\
-1.0 \\
103.0\end{array}$ & $\begin{array}{c}0.47 \\
17.9 \\
9.6 \\
8.3 \\
53.6\end{array}$ & $\begin{array}{c}0.43 \\
16.5 \\
6.8 \\
9.7 \\
41.2\end{array}$ \\
\hline $\begin{array}{l}\text { Subj. } 9(\mathrm{Ch}) \\
\text { age, } 15 \mathrm{mo} . \\
\text { wt., } 9.7 \mathrm{Kg} .\end{array}$ & 6 & $\begin{array}{l}\text { Intake } \\
\quad \text { per } \mathrm{Kg} . / \mathrm{hr} . \\
\text { total } \\
\text { Output } \\
\text { Retention } \\
\text { \% Excreted }\end{array}$ & $\begin{array}{c}25.1 \\
1,459 \\
1,035 \\
424 \\
70.9\end{array}$ & $\begin{array}{c}25.1 \\
1,459 \\
1,108 \\
351 \\
75.9\end{array}$ & $\begin{array}{c}1.81 \\
105.3 \\
23.7 \\
81.8 \\
22.5\end{array}$ & $\begin{array}{c}1.80 \\
104.9 \\
5.7 \\
99.2 \\
5.4\end{array}$ & $\begin{array}{l}0.83 \\
48.1 \\
29.4 \\
18.7 \\
61.1\end{array}$ & $\begin{array}{r}0.84 \\
48.9 \\
55.5 \\
-6.6 \\
113.5\end{array}$ & $\begin{array}{l}0.43 \\
25.1 \\
14.6 \\
10.5 \\
58.2\end{array}$ & $\begin{array}{l}0.42 \\
24.4 \\
20.3 \\
4.1 \\
83.2\end{array}$ \\
\hline $\begin{array}{c}\text { Subj. } 10(\mathrm{Ke}) \\
\text { age, } 2 \mathrm{mo} . \\
\text { wt., } 5.4 \mathrm{Kg} .\end{array}$ & 4 & $\begin{array}{l}\text { Intake } \\
\text { per } \mathrm{Kg} . / \mathrm{hr} . \\
\text { total } \\
\text { Output } \\
\text { Retention } \\
\text { \% Excreted }\end{array}$ & $\begin{array}{c}23.4 \\
506 \\
400 \\
106 \\
79.0\end{array}$ & $\begin{array}{l}23.3 \\
504 \\
333 \\
171 \\
66.1\end{array}$ & $\begin{array}{l}2.37 \\
51.3 \\
9.3 \\
42.0 \\
18.1\end{array}$ & $\begin{array}{c}2.36 \\
51.0 \\
7.8 \\
43.2 \\
15.3\end{array}$ & $\begin{array}{l}0.76 \\
16.4 \\
14.1 \\
2.3 \\
86.0\end{array}$ & $\begin{array}{c}0.77 \\
16.6 \\
24.9 \\
-8.3 \\
150.0\end{array}$ & $\begin{array}{l}0.40 \\
8.6 \\
1.9 \\
6.7 \\
22.1\end{array}$ & $\begin{array}{l}0.39 \\
8.4 \\
3.7 \\
4.7 \\
44.0\end{array}$ \\
\hline $\begin{array}{l}\text { Subj. } 11(\mathrm{Li}) \\
\text { age, } 7 \mathrm{mo} \\
\text { wt., } 6.7 \mathrm{Kg} .\end{array}$ & 6 & $\begin{array}{l}\text { Intake } \\
\quad \text { per } \mathrm{Kg} . / \mathrm{hr} . \\
\quad \text { total } \\
\text { Output } \\
\text { Retention } \\
\text { \% Excreted }\end{array}$ & $\begin{array}{c}22.3 \\
1,018 \\
858 \\
160 \\
84.3\end{array}$ & $\begin{array}{c}25.7 \\
1,033 \\
873 \\
160 \\
84.5\end{array}$ & $\begin{array}{c}2.26 \\
103.2 \\
31.8 \\
71.4 \\
30.8\end{array}$ & $\begin{array}{c}2.60 \\
104.6 \\
11.1 \\
93.2 \\
10.6\end{array}$ & $\begin{array}{c}0.72 \\
33.1 \\
36.4 \\
-3.3 \\
110.0\end{array}$ & $\begin{array}{c}0.85 \\
34.1 \\
58.6 \\
-24.5 \\
171.8\end{array}$ & $\begin{array}{l}0.38 \\
17.3 \\
13.6 \\
3.7 \\
78.6\end{array}$ & $\begin{array}{l}0.43 \\
17.2 \\
12.8 \\
4.4 \\
74.4\end{array}$ \\
\hline $\begin{array}{c}\text { Subj. } 12(\mathrm{Ch}) \\
\text { age, } 16 \mathrm{mo} \\
\text { wt., } 10.0 \mathrm{Kg} .\end{array}$ & 6 & $\begin{array}{l}\text { Intake } \\
\quad \text { per } \mathrm{Kg} . / \mathrm{hr} . \\
\quad \text { total } \\
\text { Output } \\
\text { Retention } \\
\text { \% Excreted }\end{array}$ & & $\begin{array}{c}25.6 \\
1,534 \\
1,101 \\
433 \\
71.8\end{array}$ & & $\begin{array}{c}2.59 \\
155.4 \\
24.2 \\
131.2 \\
15.6\end{array}$ & & $\begin{array}{c}0.84 \\
50.6 \\
91.4 \\
-40.8 \\
180.6\end{array}$ & & $\begin{array}{c}0.43 \\
25.6 \\
18.8 \\
6.8 \\
73.4\end{array}$ \\
\hline $\begin{array}{l}\text { Average* } \\
\text { age, } 6.5 \mathrm{mo} \text {. } \\
\text { Wt., } 6.8 \mathrm{Kg} \text {. }\end{array}$ & & $\begin{array}{l}\text { Intake } \\
\text { per Kg./hr. } \\
\text { \% Excreted }\end{array}$ & $\begin{array}{l}24.9 \\
78.9\end{array}$ & $\begin{array}{r}24.9 \\
p>0.9 \\
80.7 \\
p>0.6\end{array}$ & $\begin{array}{r}1.96 \\
20.0\end{array}$ & $\begin{aligned} & 1.97 \\
& >0.8 \\
& 9.87 \dagger \\
p & <0.02\end{aligned}$ & 79.0 & $\begin{array}{c}\quad 0.83 \\
p>0.5 \\
126.7 \dagger \\
p<0.01\end{array}$ & ${ }_{53.1}^{0.43} \mathrm{p}$ & $\begin{aligned} & 0.41 \\
&> 0.3 \\
& 58.6 \\
&>0.3\end{aligned}$ \\
\hline
\end{tabular}

* Averages excluding Subject $12 . \quad$ † Significant differences. 
lower than that noted with fructose, 127 per cent $(\mathrm{p}<0.01)$.

There were no significant differences related to the sugar infused in the intakes of water, sodium, potassium and hexose, nor in the percentages of the potassium and water intakes excreted in the urine.

The average blood levels for glucose and fructose at two hourly intervals during infusion and one hour postinfusion are illustrated in Figure 1. In agreement with the findings of other observers $(1,3)$ levels of total reducing substance were found to be significantly higher during glucose infusion. The concentrations tended to fall during the course of infusion of both sugars although the tendency was more marked in the case of fructose and especially so at the higher rate of infusion.

The only significant alteration in blood electrolytes was noted in the fructose infusions in Group $B$ and consisted of a fall in serum sodium from a mean initial level of 149 to a final level of 140 $\mathrm{mEq}$. per L. This finding correlates with the negative sodium balance noted in these subjects. Variations in hematocrit were without significance in either group.

No significant alterations in pulse and respiratory rates and liver size were noted during the infusions to the subjects in Group A. The average values for these measurements at the start and end of the infusions administered to the subjects in Group B are given in Table IV. Statistically significant changes were noted in all three only during the fructose infusion. The alterations ob- served were attributed to differences in the metabolism of fructose, because the rates of administration of water, electrolytes and carbohydrates were the same in the experiments with both sugars.

During the latter part of a fructose infusion in Subject 12, administered at the very rapid rate of $2.59 \mathrm{Gm}$. per $\mathrm{Kg}$. per hour, marked hyperventilation, tachycardia, pallor and mottling appeared. Blood was drawn in an effort to determine whether this reaction was the result of circulatory or metabolic causes. The $\mathrm{pH}$ of venous blood was found to be 6.85 and the $\mathrm{CO}_{2}$ content $5.5 \mathrm{mM}$ per $\mathrm{L}$. One hour after termination of the infusion, the levels had increased to 7.02 and $13 \mathrm{mM}$ per L., respectively, coincident with marked improvement in the patient's condition. Because of the severity of the disturbance accompanying rapid fructose infusion in this subject, it did not seem justifiable to subject him to further study by administering a comparable glucose infusion. Nevertheless; the possibility suggested itself that the significant rises in pulse and respiratory rate which occurred only during fructose infusion might reflect the presence of a similar metabolic acidosis in the group of subjects infused, at a rapid rate, with this carbohydrate. Further investigation, bearing on this question, was undertaken.

\section{Study of acid-base changes}

Complete data on the blood levels found and differences existing between initial and final blood levels in each subject studied are recorded in

TABLE IV

$P$ ulse and respiration rates and liver size at beginning and end of infusion-Subjects in Group B

\begin{tabular}{|c|c|c|c|c|c|c|c|c|c|c|c|c|}
\hline \multirow[b]{3}{*}{ Subject } & \multicolumn{4}{|c|}{ Pulse rate } & \multicolumn{4}{|c|}{ Respiratory rate } & \multicolumn{4}{|c|}{ Liver size* } \\
\hline & \multicolumn{2}{|c|}{ Glucose } & \multicolumn{2}{|c|}{ Fructose } & \multicolumn{2}{|c|}{ Glucose } & \multicolumn{2}{|c|}{ Fructose } & \multicolumn{2}{|c|}{ Glucose } & \multicolumn{2}{|c|}{ Fructose } \\
\hline & I† & $\overline{F \ddagger}$ & I & F & $I$ & $\mathbf{F}$ & I & F & I & $\mathbf{F}$ & I & F \\
\hline $\begin{array}{r}4 \\
5 \\
6 \\
7 \\
8 \\
9 \\
10 \\
11\end{array}$ & $\begin{array}{r}150 \\
98 \\
122 \\
140 \\
120 \\
124 \\
160 \\
120\end{array}$ & $\begin{array}{l}124 \\
106 \\
132 \\
132 \\
120 \\
120 \\
200 \\
160\end{array}$ & $\begin{array}{r}90 \\
110 \\
144 \\
140 \\
128 \\
146 \\
128 \\
156\end{array}$ & $\begin{array}{l}124 \\
160 \\
159 \\
144 \\
128 \\
152 \\
160 \\
160\end{array}$ & $\begin{array}{l}36 \\
24 \\
36 \\
40 \\
40 \\
28 \\
36\end{array}$ & $\begin{array}{l}36 \\
32 \\
28 \\
30 \\
28 \\
32 \\
40\end{array}$ & $\begin{array}{l}32 \\
36 \\
38 \\
50 \\
36 \\
32 \\
32 \\
32\end{array}$ & $\begin{array}{l}58 \\
44 \\
38 \\
70 \\
64 \\
32 \\
48 \\
42\end{array}$ & $\begin{array}{r}3.0 \\
2.0 \\
1.0 \\
1.5 \\
2.0 \\
2.5 \\
0 \\
2.0\end{array}$ & $\begin{array}{l}2.0 \\
2.0 \\
2.0 \\
1.5 \\
2.0 \\
3.0 \\
1.5 \\
2.5\end{array}$ & $\begin{array}{r}2.0 \\
0 \\
1.0 \\
2.0 \\
2.0 \\
0.5 \\
1.0 \\
2.0\end{array}$ & $\begin{array}{l}2.0 \\
2.0 \\
3.0 \\
3.0 \\
3.0 \\
4.5 \\
3.0 \\
3.5\end{array}$ \\
\hline $\begin{array}{c}\text { Mean } \\
\text { p }\end{array}$ & \multicolumn{2}{|c|}{${ }^{129}>0.3^{137}$} & \multicolumn{2}{|c|}{${ }_{<0.05}^{130}$} & \multicolumn{2}{|c|}{$\stackrel{34}{>} 0.7^{32}$} & \multicolumn{2}{|c|}{$\begin{array}{c}36 \\
<0.01\end{array}$} & \multicolumn{2}{|c|}{$1.7>0.2^{2.1}$} & \multicolumn{2}{|c|}{${ }^{1.3}<0.01^{3.0}$} \\
\hline
\end{tabular}

* Measured as cm. palpable below costal margin at midclavicular line.

$\dagger$ Initial.

$\ddagger$ Final. 
STUDY OF GLUCOSE AND FRUCTOSE METABOLISM IN INFANTS

TABLE V

Blood analyses during hexose infusion

\begin{tabular}{|c|c|c|c|c|c|c|c|c|}
\hline Subject & $\begin{array}{l}\text { Time } \\
\text { hrs. }\end{array}$ & $\mathrm{pH}$ & $\underset{m E q . / L}{\mathrm{CO}_{2}}$ & $\underset{m E q . / L}{\mathrm{Na}}$ & $\underset{m E q . / L}{\mathbf{K}}$ & $\underset{m E q . / L .}{\mathrm{Cl}}$ & $\begin{array}{l}\text { Lactate } \\
m E q . / L\end{array}$ & $\begin{array}{c}\text { Pyruvate } \\
m E q . / L \text {. }\end{array}$ \\
\hline \multicolumn{9}{|c|}{ Fructose infusion } \\
\hline R. Ro. & $\begin{array}{l}0 \\
4 \\
\Delta\end{array}$ & $\begin{array}{r}7.30 \\
7.29 \\
-0.01\end{array}$ & $\begin{array}{r}18.3 \\
14.6 \\
-3.7\end{array}$ & $\begin{array}{l}136 \\
130 \\
-6\end{array}$ & $\begin{array}{r}5.3 \\
5.7 \\
+0.4\end{array}$ & $\begin{array}{l}104 \\
102 \\
-2\end{array}$ & $\begin{array}{l}1.4 \\
3.8 \\
2.4\end{array}$ & $\begin{array}{l}0.2 \\
0.3 \\
0.1\end{array}$ \\
\hline V. Lo. & $\begin{array}{l}0 \\
4 \\
6 \\
\Delta\end{array}$ & $\begin{array}{r}7.33 \\
7.12 \\
7.11 \\
-0.22\end{array}$ & $\begin{array}{r}18.8 \\
9.1 \\
7.9 \\
-10.9\end{array}$ & $\begin{array}{r}140 \\
119 \\
125 \\
-15\end{array}$ & $\begin{array}{r}4.6 \\
5.7 \\
6.5 \\
+1.9\end{array}$ & $\begin{array}{l}105 \\
104 \\
101 \\
-4\end{array}$ & $\begin{array}{l}1.5 \\
6.7 \\
7.0 \\
5.5\end{array}$ & $\begin{array}{l}0.2 \\
0.5 \\
0.5 \\
0.3\end{array}$ \\
\hline L. Gl. & $\begin{array}{l}0 \\
4 \\
6 \\
\Delta\end{array}$ & $\begin{array}{r}7.38 \\
7.28 \\
7.24 \\
-0.14\end{array}$ & $\begin{array}{r}19.4 \\
14.9 \\
14.6 \\
-4.8\end{array}$ & $\begin{array}{r}141 \\
131 \\
126 \\
-15\end{array}$ & $\begin{array}{r}5.0 \\
6.5 \\
6.2 \\
+1.2\end{array}$ & $\begin{array}{l}104 \\
102 \\
103 \\
-1\end{array}$ & $\begin{array}{l}1.4 \\
3.5 \\
4.3 \\
2.9\end{array}$ & $\begin{array}{l}0.1 \\
0.3 \\
0.4 \\
0.3\end{array}$ \\
\hline R. Or. & $\begin{array}{l}0 \\
6 \\
\Delta\end{array}$ & $\begin{array}{r}7.31 \\
7.20 \\
-0.11\end{array}$ & $\begin{array}{r}18.7 \\
13.0 \\
-5.7\end{array}$ & $\begin{array}{r}139 \\
124 \\
-15\end{array}$ & 4.3 & 107 & $\begin{array}{l}1.4 \\
5.7 \\
4.3\end{array}$ & $\begin{array}{l}0.2 \\
0.4 \\
0.2\end{array}$ \\
\hline R. Ze. & $\begin{array}{l}0 \\
4 \\
\Delta\end{array}$ & $\begin{array}{r}7.41 \\
7.29 \\
-0.12\end{array}$ & $\begin{array}{r}18.2 \\
11.6 \\
-6.6\end{array}$ & $\begin{array}{r}135 \\
118 \\
-17\end{array}$ & $\begin{array}{r}4.8 \\
4.7 \\
-0.1\end{array}$ & $\begin{array}{r}101 \\
98 \\
-3\end{array}$ & $\begin{array}{l}1.6 \\
6.4 \\
4.8\end{array}$ & $\begin{array}{l}0.2 \\
0.5 \\
0.3\end{array}$ \\
\hline D. La. & $\begin{array}{l}0 \\
4 \\
6 \\
\Delta\end{array}$ & $\begin{array}{r}7.42 \\
7.32 \\
7.28 \\
-0.14\end{array}$ & $\begin{array}{r}19.1 \\
12.5 \\
11.5 \\
-7.6\end{array}$ & $\begin{array}{r}140 \\
129 \\
121 \\
-19\end{array}$ & $\begin{array}{r}4.8 \\
5.0 \\
4.7 \\
-0.1\end{array}$ & $\begin{array}{r}104 \\
101 \\
99 \\
-5\end{array}$ & $\begin{array}{l}1.4 \\
6.0 \\
5.4 \\
4.0\end{array}$ & $\begin{array}{l}0.2 \\
0.4 \\
0.5 \\
0.3\end{array}$ \\
\hline R. Ma. & $\begin{array}{l}0 \\
4 \\
6 \\
\Delta\end{array}$ & $\begin{array}{r}7.35 \\
7.25 \\
7.21 \\
-0.14\end{array}$ & $\begin{array}{r}19.3 \\
11.9 \\
10.5 \\
-8.8\end{array}$ & $\begin{array}{r}140 \\
135 \\
129 \\
-11\end{array}$ & $\begin{array}{l}4.4 \\
4.8\end{array}$ & $\begin{array}{l}101 \\
102 \\
100 \\
-1\end{array}$ & $\begin{array}{l}1.4 \\
5.4 \\
5.2 \\
3.8\end{array}$ & $\begin{array}{l}0.2 \\
0.3 \\
0.3 \\
0.1\end{array}$ \\
\hline & $\mathrm{m}$ & & -6.8 & -14 & +0.66 & -2.7 & 3.96 & 0.24 \\
\hline \multicolumn{9}{|c|}{ Glucose infusion } \\
\hline D. Ga. & $\begin{array}{l}0 \\
4 \\
6 \\
\Delta\end{array}$ & $\begin{array}{r}7.38 \\
7.29 \\
7.32 \\
-0.06\end{array}$ & $\begin{array}{r}19.1 \\
19.9 \\
22.6 \\
+3.5\end{array}$ & $\begin{array}{l}137 \\
132 \\
133 \\
-4\end{array}$ & $\begin{array}{r}4.2 \\
3.8 \\
4.6 \\
+0.4\end{array}$ & $\begin{array}{l}103 \\
100 \\
100 \\
-3\end{array}$ & $\begin{array}{l}1.2 \\
2.8 \\
3.9 \\
2.7\end{array}$ & $\begin{array}{l}0.2 \\
0.3 \\
0.4 \\
0.2\end{array}$ \\
\hline P. Ya. & $\begin{array}{l}0 \\
4 \\
6 \\
\Delta\end{array}$ & $\begin{array}{r}7.36 \\
7.38 \\
7.38 \\
+0.02\end{array}$ & $\begin{array}{r}17.8 \\
15.8 \\
17.0 \\
-0.8\end{array}$ & $\begin{array}{r}137 \\
128 \\
126 \\
-11\end{array}$ & $\begin{array}{r}4.6 \\
4.2 \\
4.0 \\
-0.6\end{array}$ & $\begin{array}{r}100 \\
99 \\
98 \\
-2\end{array}$ & $\begin{array}{l}1.0 \\
2.1 \\
2.2 \\
1.2\end{array}$ & $\begin{array}{r}0.2 \\
0.3 \\
0.3 \\
+0.1\end{array}$ \\
\hline L. ${ }_{3}^{\mathrm{Hi}}$ & $\begin{array}{l}0 \\
4 \\
6 \\
\Delta\end{array}$ & $\begin{array}{r}7.39 \\
7.38 \\
7.37 \\
-0.02\end{array}$ & $\begin{array}{r}18.5 \\
16.5 \\
15.7 \\
-2.8\end{array}$ & $\begin{array}{l}137 \\
134 \\
131 \\
-6\end{array}$ & $\begin{array}{r}5.3 \\
4.7 \\
4.8 \\
-0.5\end{array}$ & $\begin{array}{r}100 \\
98 \\
101 \\
+1\end{array}$ & $\begin{array}{l}1.2 \\
4.1 \\
3.9 \\
2.7\end{array}$ & $\begin{array}{l}0.2 \\
0.4 \\
0.4 \\
0.2\end{array}$ \\
\hline$\underset{4}{\mathrm{~J} . \mathrm{Ga}}$ & $\begin{array}{l}0 \\
4 \\
6 \\
\Delta\end{array}$ & $\begin{array}{r}7.40 \\
7.37 \\
7.36 \\
-0.04\end{array}$ & $\begin{array}{r}19.0 \\
18.6 \\
18.9 \\
-0.1\end{array}$ & $\begin{array}{r}138 \\
129 \\
126 \\
-12\end{array}$ & $\begin{array}{r}3.8 \\
3.8 \\
3.9 \\
+0.1\end{array}$ & $\begin{array}{r}103 \\
99 \\
100 \\
-3\end{array}$ & $\begin{array}{l}1.5 \\
2.3 \\
2.9 \\
1.4\end{array}$ & $\begin{array}{l}0.2 \\
0.2 \\
0.3 \\
0.1\end{array}$ \\
\hline D. Da. & $\begin{array}{l}0 \\
6 \\
\Delta\end{array}$ & $\begin{array}{r}7.40 \\
7.37 \\
-0.03\end{array}$ & $\begin{array}{r}19.3 \\
18.2 \\
-1.1\end{array}$ & $\begin{array}{r}139 \\
135 \\
-4\end{array}$ & 5.4 & $\begin{array}{r}101 \\
101 \\
0\end{array}$ & $\begin{array}{l}1.4 \\
2.1 \\
0.7\end{array}$ & $\begin{array}{l}0.2 \\
0.3 \\
0.1\end{array}$ \\
\hline R. $\underset{6}{\operatorname{Pr}}$ & $\begin{array}{l}0 \\
4 \\
6 \\
\Delta\end{array}$ & $\begin{array}{r}7.40 \\
7.37 \\
7.38 \\
-0.02\end{array}$ & $\begin{array}{r}19.3 \\
19.1 \\
18.5 \\
-0.8\end{array}$ & $\begin{array}{r}139 \\
137 \\
135 \\
-4\end{array}$ & $\begin{array}{r}3.9 \\
3.8 \\
3.7 \\
-0.2\end{array}$ & $\begin{array}{l}102 \\
102 \\
103 \\
+1\end{array}$ & $\begin{array}{l}1.4 \\
3.8 \\
3.9 \\
2.5\end{array}$ & $\begin{array}{l}0.1 \\
0.3 \\
0.3 \\
0.2\end{array}$ \\
\hline E. Ca. & $\begin{array}{l}0 \\
4 \\
6 \\
\Delta\end{array}$ & $\begin{array}{r}7.41 \\
7.37 \\
7.40 \\
-0.01\end{array}$ & $\begin{array}{r}19.1 \\
18.5 \\
18.7 \\
-0.4\end{array}$ & $\begin{array}{l}137 \\
135 \\
131 \\
-6\end{array}$ & 3.7 & $\begin{array}{l}103 \\
101 \\
101 \\
-2\end{array}$ & $\begin{array}{l}1.4 \\
2.2 \\
2.2 \\
0.8\end{array}$ & $\begin{array}{l}0.1 \\
0.2 \\
0.2 \\
0.1\end{array}$ \\
\hline & $\mathrm{m}$ & -0.02 & -0.4 & -6.7 & 0.16 & -1.1 & 1.70 & 0.11 \\
\hline & $\mathbf{p}$ & $<0.01$ & $<0.001$ & $<0.01$ & $>0.1$ & $>0.1$ & $<0.01$ & $<0.01$ \\
\hline
\end{tabular}


Table V. The means of the changes in each of the blood levels have been calculated separately for the group of subjects receiving fructose and those receiving glucose. The significance of the difference in these means is recorded in Table V.

The data demonstrate significantly greater rises in blood lactic and pyruvic acids in the subjects receiving fructose, than in those infused with glu- cose. The mean increases in lactate were 35.6 mg. per cent ( $3.96 \mathrm{mEq}$. per L.) and $15.3 \mathrm{mg}$. per cent $(1.71 \mathrm{mEq}$. per L.) for the fructose and glucose groups, respectively. Blood pyruvate rose an average of $2.16 \mathrm{mg}$. per cent $(0.24 \mathrm{mEq}$. per L.) with fructose and $0.98 \mathrm{mg}$. per cent $(0.11$ $\mathrm{mEq}$. per L.) with glucose. Accompanying the acid metabolite accumulation noted with fructose

TABLE VI

Balance data

\begin{tabular}{|c|c|c|c|c|c|c|c|c|}
\hline Subject & $\begin{array}{c}\text { Infusion } \\
\text { time } \\
\text { hrs. }\end{array}$ & & $\underset{m l .}{\mathrm{H}_{2} \mathrm{O}}$ & $\underset{\mathrm{Gm} .}{\mathrm{CHO}}$ & $\underset{m E q}{\mathrm{Na}}$ & $\underset{m E q}{\mathbf{K}}$ & $\begin{array}{c}\text { Lactate } \\
m E q .\end{array}$ & $\begin{array}{l}\text { Pyruvate } \\
m E q .\end{array}$ \\
\hline \multicolumn{9}{|c|}{ Fructose infusion } \\
\hline $\begin{array}{l}\text { R. Ro. } \\
\text { Male } \\
6 \text { mos. } \\
7.2 \mathrm{Kg} .\end{array}$ & 4 & $\begin{array}{l}\text { Intake } \\
\text { per } \mathrm{Kg} . / \mathrm{hr} . \\
\text { total } \\
\text { Output } \\
\text { Retention } \\
\text { \% Excreted }\end{array}$ & $\begin{array}{l}22.1 \\
638 \\
322 \\
316 \\
50.5\end{array}$ & $\begin{array}{r}2.0 \\
57.8 \\
6.8 \\
51.0 \\
11.8\end{array}$ & $\begin{array}{c}0.60 \\
17.3 \\
15.0 \\
2.3 \\
86.7\end{array}$ & $\begin{array}{l}0.30 \\
8.7 \\
8.3 \\
0.4 \\
95.4\end{array}$ & 0.96 & 0.17 \\
\hline $\begin{array}{l}\text { V. Lo. } \\
\text { Female } \\
8 \text { mos. } \\
8.5 \mathrm{Kg} .\end{array}$ & 6 & $\begin{array}{l}\text { Intake } \\
\text { per Kg./hr. } \\
\text { total } \\
\text { Output } \\
\text { Retention } \\
\text { \% Excreted }\end{array}$ & $\begin{array}{c}20.3 \\
1,034 \\
737 \\
297 \\
71.3\end{array}$ & $\begin{array}{c}2.03 \\
103.4 \\
9.9 \\
93.5 \\
9.6\end{array}$ & $\begin{array}{r}0.61 \\
31.0 \\
42.0 \\
-11.0 \\
135.5\end{array}$ & $\begin{array}{c}0.30 \\
15.5 \\
8.9 \\
6.6 \\
57.4\end{array}$ & 6.83 & 0.52 \\
\hline $\begin{array}{l}\text { L. Gl. } \\
\text { Female } \\
2 \text { yrs. } \\
12.7 \mathrm{Kg} .\end{array}$ & 6 & $\begin{array}{l}\text { Intake } \\
\quad \text { per } \mathrm{Kg} . / \mathrm{hr} . \\
\text { total } \\
\text { Output } \\
\text { Retention } \\
\text { \% Excreted }\end{array}$ & $\begin{array}{c}19.6 \\
1,492 \\
1,022 \\
470 \\
68.5\end{array}$ & $\begin{array}{c}1.96 \\
149.2 \\
11.6 \\
137.6 \\
7.8\end{array}$ & $\begin{array}{c}0.59 \\
44.8 \\
49.2 \\
-4.4 \\
109.8\end{array}$ & $\begin{array}{l}0.29 \\
22.4 \\
11.3 \\
11.1 \\
50.4\end{array}$ & 5.72 & 0.99 \\
\hline $\begin{array}{l}\text { R. Or. } \\
\text { Female } \\
5 \text { mos. } \\
6.0 \mathrm{Kg} .\end{array}$ & 6 & $\begin{array}{l}\text { Intake } \\
\quad \text { per } \mathrm{Kg} . / \mathrm{hr} . \\
\text { total } \\
\text { Output } \\
\text { Retention } \\
\text { \% Excreted }\end{array}$ & $\begin{array}{c}20.0 \\
721 \\
508 \\
213 \\
70.4\end{array}$ & $\begin{array}{r}2.0 \\
72.1 \\
7.4 \\
64.7 \\
10.3\end{array}$ & $\begin{array}{c}0.60 \\
21.6 \\
20.5 \\
+1.1 \\
94.9\end{array}$ & $\begin{array}{r}0.30 \\
10.8 \\
8.8 \\
+2.0 \\
81.5\end{array}$ & 4.38 & 0.56 \\
\hline $\begin{array}{l}\text { R. Ze. } \\
\text { Male } \\
3 \text { mos. } \\
5.7 \mathrm{Kg} \text {. }\end{array}$ & 4.25 & $\begin{array}{l}\text { Intake } \\
\quad \text { per Kg./hr. } \\
\text { total } \\
\text { Output } \\
\text { Retention } \\
\text { \% Excreted }\end{array}$ & $\begin{array}{l}19.4 \\
470 \\
302 \\
168 \\
64.2\end{array}$ & $\begin{array}{c}1.94 \\
47.0 \\
2.6 \\
44.4 \\
5.5\end{array}$ & $\begin{array}{c}0.58 \\
14.1 \\
12.5 \\
1.6 \\
88.6\end{array}$ & $\begin{array}{l}0.29 \\
7.0 \\
4.3 \\
2.7 \\
61.4\end{array}$ & 1.69 & 0.25 \\
\hline $\begin{array}{l}\text { D. La. } \\
\text { Male } \\
2.5 \mathrm{mos} . \\
5.5 \mathrm{Kg} \text {. }\end{array}$ & 6 & $\begin{array}{l}\text { Intake } \\
\quad \text { per Kg./hr. } \\
\text { total } \\
\text { Output } \\
\text { Retention } \\
\text { \% Excreted }\end{array}$ & $\begin{array}{c}20.4 \\
675 \\
486 \\
189 \\
72.0\end{array}$ & $\begin{array}{c}2.04 \\
67.5 \\
6.7 \\
60.8 \\
9.9\end{array}$ & $\begin{array}{c}0.61 \\
20.2 \\
16.1 \\
4.1 \\
79.7\end{array}$ & $\begin{array}{c}0.31 \\
10.1 \\
6.2 \\
3.9 \\
61.4\end{array}$ & 7.43 & 0.63 \\
\hline $\begin{array}{l}\text { R. Ma. } \\
\text { Male } \\
6 \text { mos. } \\
6.9 \mathrm{Kg} .\end{array}$ & 6 & $\begin{array}{l}\text { Intake } \\
\quad \text { per Kg./hr. } \\
\text { total } \\
\text { Output } \\
\text { Retention } \\
\text { \% Excreted }\end{array}$ & $\begin{array}{c}20.5 \\
848 \\
527 \\
321 \\
62.1\end{array}$ & $\begin{array}{c}2.05 \\
84.8 \\
4.4 \\
80.4 \\
5.2\end{array}$ & $\begin{array}{c}0.61 \\
25.4 \\
25.3 \\
+0.1 \\
99.6\end{array}$ & $\begin{array}{c}0.31 \\
12.7 \\
6.4 \\
6.3 \\
50.4\end{array}$ & 6.20 & 0.44 \\
\hline $\mathbf{m}$ & . & $\begin{array}{l}\text { Intake } \\
\text { per Kg./hr. } \\
\% \text { Excreted }\end{array}$ & $\begin{array}{l}20.3 \\
65.6\end{array}$ & $\begin{array}{l}2.00 \\
8.6\end{array}$ & $\begin{array}{c}0.60 \\
99.2\end{array}$ & $\begin{array}{l}0.30 \\
65.4\end{array}$ & & \\
\hline
\end{tabular}




\begin{tabular}{|c|c|c|c|c|c|c|c|c|}
\hline Subject & $\begin{array}{c}\text { Infusion } \\
\text { time } \\
\text { hrs. }\end{array}$ & & $\underset{m l .}{\mathrm{H}_{2} \mathrm{O}}$ & $\underset{G m .}{\mathrm{CHO}}$ & $\underset{m E q}{\mathrm{Na}}$ & $\underset{m E q}{\mathrm{~K}}$ & $\begin{array}{l}\text { Lactate } \\
m E q .\end{array}$ & $\begin{array}{c}\text { Pyruvate } \\
m E q .\end{array}$ \\
\hline \multicolumn{9}{|c|}{ Glucose infusion } \\
\hline $\begin{array}{l}\text { Female } \\
11 \mathrm{mos} \text {. } \\
8.1 \mathrm{Kg} .\end{array}$ & 0 & $\begin{array}{l}\text { per Kg./hr. } \\
\text { total } \\
\text { Output } \\
\text { Retention } \\
\text { \% Excreted }\end{array}$ & $\begin{array}{c}21.6 \\
1,049 \\
691 \\
358 \\
65.9\end{array}$ & $\begin{array}{l}1.94 \\
94.3 \\
10.2 \\
84.1 \\
11.9\end{array}$ & $\begin{array}{c}0.62 \\
30.0 \\
25.6 \\
4.4 \\
85.3\end{array}$ & $\begin{array}{c}0.30 \\
14.7 \\
6.6 \\
8.1 \\
44.9\end{array}$ & 0.32 & 0.24 \\
\hline $\begin{array}{l}\text { P. Ya. } \\
\text { Female } \\
18 \text { mos. } \\
9.1 \mathrm{Kg} .\end{array}$ & 6 & $\begin{array}{l}\text { Intake } \\
\quad \text { per } \mathrm{Kg} . / \mathrm{hr} . \\
\text { total } \\
\text { Output } \\
\text { Retention } \\
\text { \% Excreted }\end{array}$ & $\begin{array}{c}19.8 \\
1,083 \\
748 \\
335 \\
69.1\end{array}$ & $\begin{array}{c}1.98 \\
108.3 \\
44.2 \\
64.1 \\
40.8\end{array}$ & $\begin{array}{c}0.61 \\
33.6 \\
24.7 \\
8.9 \\
73.5\end{array}$ & $\begin{array}{c}0.31 \\
16.9 \\
17.7 \\
-0.8 \\
104.7\end{array}$ & 0.21 & 0.10 \\
\hline $\begin{array}{l}\text { L. Hi. } \\
\text { Male } \\
2.5 \mathrm{mos} . \\
5.3 \mathrm{Kg} .\end{array}$ & 6 & $\begin{array}{l}\text { Intake } \\
\quad \text { per } \mathrm{Kg} . / \mathrm{hr} . \\
\quad \text { total } \\
\text { Output } \\
\text { Retention } \\
\text { \% Excreted }\end{array}$ & $\begin{array}{c}20.2 \\
643 \\
491 \\
162 \\
74.8\end{array}$ & $\begin{array}{l}2.10 \\
66.7 \\
12.8 \\
53.4 \\
19.2\end{array}$ & $\begin{array}{c}0.62 \\
19.6 \\
20.9 \\
-1.3 \\
106.6\end{array}$ & $\begin{array}{l}0.30 \\
9.6 \\
4.4 \\
5.2 \\
45.8\end{array}$ & 0.54 & 0.15 \\
\hline $\begin{array}{l}\text { J. Ga. } \\
\text { Male } \\
7 \text { mos. } \\
9.15 \mathrm{Kg} .\end{array}$ & 6 & $\begin{array}{l}\text { Intake } \\
\quad \text { per } \mathrm{Kg} . / \mathrm{hr} . \\
\quad \text { total } \\
\text { Output } \\
\text { Retention } \\
\text { \% Excreted }\end{array}$ & $\begin{array}{c}19.9 \\
1,094 \\
753 \\
341 \\
68.8\end{array}$ & $\begin{array}{l}1.78 \\
98.0 \\
28.6 \\
69.4 \\
29.2\end{array}$ & & $\begin{array}{c}0.31 \\
17.1 \\
6.0 \\
11.1 \\
35.1\end{array}$ & 0.49 & 0.29 \\
\hline $\begin{array}{l}\text { D. Da. } \\
\text { Male } \\
8 \text { mos. } \\
7.0 \mathrm{Kg} .\end{array}$ & 6 & $\begin{array}{l}\text { Intake } \\
\quad \text { per } \mathrm{Kg} . / \mathrm{hr} . \\
\quad \text { total } \\
\text { Output } \\
\text { Retention } \\
\text { \% Excreted }\end{array}$ & $\begin{array}{c}19.6 \\
823 \\
487 \\
336 \\
59.4\end{array}$ & $\begin{array}{l}1.80 \\
75.6 \\
14.0 \\
61.6 \\
18.5\end{array}$ & $\begin{array}{c}0.61 \\
25.7 \\
12.4 \\
13.3 \\
48.2\end{array}$ & $\begin{array}{c}0.30 \\
12.7 \\
4.7 \\
8.0 \\
37.0\end{array}$ & 0.16 & 0.15 \\
\hline $\begin{array}{l}\text { R. Py } \\
\text { Male } \\
14 \text { mos. } \\
11.4 \mathrm{Kg} .\end{array}$ & 6 & $\begin{array}{l}\text { Intake } \\
\quad \text { per } \mathrm{Kg} . / \mathrm{hr} . \\
\quad \text { total } \\
\text { Output } \\
\text { Retention } \\
\text { \% Excreted }\end{array}$ & $\begin{array}{c}19.9 \\
1,362 \\
962 \\
400 \\
70.6\end{array}$ & $\begin{array}{c}1.93 \\
132.6 \\
21.2 \\
111.6 \\
16.0\end{array}$ & $\begin{array}{c}0.62 \\
42.2 \\
34.6 \\
7.6 \\
82.0\end{array}$ & $\begin{array}{c}0.30 \\
20.8 \\
6.5 \\
14.3 \\
31.2\end{array}$ & 0.54 & 0.21 \\
\hline $\begin{array}{l}\text { E. Ca. } \\
\text { Male } \\
8 \text { mos. } \\
8.6 \mathrm{Kg} .\end{array}$ & 6 & $\begin{array}{l}\text { Intake } \\
\quad \text { per } \mathrm{Kg} . / \mathrm{hr} . \\
\quad \text { total } \\
\text { Output } \\
\text { Retention } \\
\text { \% Excreted }\end{array}$ & $\begin{array}{c}20.1 \\
1,038 \\
801 \\
237 \\
77.2\end{array}$ & $\begin{array}{c}1.99 \\
102.8 \\
30.8 \\
72.0 \\
30.0\end{array}$ & $\begin{array}{c}0.62 \\
32.2 \\
29.6 \\
2.6 \\
91.9\end{array}$ & $\begin{array}{c}0.31 \\
16.2 \\
11.9 \\
4.3 \\
73.4\end{array}$ & 0.15 & 0.12 \\
\hline $\begin{array}{l}\mathbf{m} \\
\mathbf{m}\end{array}$ & & $\begin{array}{l}\text { Intake } \\
\text { per Kg./hr. } \\
\text { \% Excreted }\end{array}$ & $\begin{array}{l}20.2 \\
69.4\end{array}$ & $\begin{array}{c}1.94 \\
23.6\end{array}$ & $\begin{array}{l}0.62 \\
81.2\end{array}$ & $\begin{array}{c}0.30 \\
53.1\end{array}$ & & \\
\hline $\mathbf{p}$ & & & $>0.3$ & $<0.01$ & $>0.1$ & $>0.3$ & $<0.001$ & $<0.01$ \\
\hline
\end{tabular}

infusion, decreases in blood $\mathrm{pH}$ ranging from 0.11 to 0.22 were seen in six of the seven subjects, and mean decreases in whole blood $\mathrm{CO}_{2}$ of $6.8 \mathrm{mM}$ per L. (range, 3.7 to $10.0 \mathrm{mM}$ per L.), and in plasma sodium of $14 \mathrm{mEq}$. per L. (range, 6 to 19 $\mathrm{mEq}$. per L.) were found. These values likewise differ significantly from those noted in the glucose group, where decreases in blood $\mathrm{pH}$ ranged from
0.01 to 0.06 and mean decreases in $\mathrm{CO}_{2}$ of $0.4 \mathrm{mM}$ per L. (range, -2.8 to $+3.5 \mathrm{mM}$ per L.), and in sodium of $6.7 \mathrm{mEq}$. per L. (range, 4 to 12 ) were seen. No significant differences in potassium or chloride levels were demonstrated.

Table VI presents data for intake and excretion of water, carbohydrate, sodium and potassium, as well as excretion of lactate and pyruvate of the 
individual subjects. As in the previous study, the excretion of fructose (8.6 per cent of intake) was significantly lower than that of glucose $(23.6 \mathrm{per}$ cent), but in contrast to the earlier group of subjects, the children who received fructose in general did not show a negative sodium balance. The mean sodium excretion of the individuals receiving fructose was higher ( 99.2 per cent of intake) than that noted with the glucose infusions (81.2 per cent), but not significantly so. The greater mean sodium loss noted in the first study probably reflects the very high infusion rates used in several of the subjects. As before, no significant difference was seen in water or potassium excretions with the two carbohydrates.

A highly significant difference was found in the lactic acid excretions of the two groups, $10.4 \mathrm{mg}$. per $\mathrm{Kg}$. per hour and $0.6 \mathrm{mg}$. per $\mathrm{Kg}$. per hour for the subjects receiving fructose and glucose, respectively. A significant but less striking difference existed in pyruvate excretion, which averaged $0.6 \mathrm{mg}$. per $\mathrm{Kg}$. per hour with fructose administration and $0.3 \mathrm{mg}$. per $\mathrm{Kg}$. per hour with glucose.

\section{DISCUSSION}

The results reported here established the fact that infants can utilize fructose almost completely when it is administered at the relatively rapid rate of $1.0 \mathrm{Gm}$. per $\mathrm{Kg}$. per hour for periods of at least six hours. This rate is equivalent to the maximum set for glucose by Butler and Talbot (7) and confirmed for infants in the present study. When the two sugars were administered at an average rate of $2.0 \mathrm{Gm}$. per $\mathrm{Kg}$. per hour in order to demonstrate a possible superiority in fructose utilization, there was a statistically significant difference in utilization in favor of fructose.

As stated above, there were indications that the superior utilization of fructose, at rapid rates of infusion, was not entirely advantageous. Statistically significant increments in pulse and respiratory rate were demonstrated during fructose infusion, and appeared to be manifestations of cardiorespiratory responses to an altered metabolic state. A significant enlargement of the liver was likewise noted in association with rapid fructose infusion, probably as the result of the more rapid uptake $(17,18)$ and conversion of this sugar to glycogen (19). In addition, the subjects in Group B ex- hibited an average negative sodium balance with fructose infusion but not. with glucose. This difference was not noted in the subsequent series of experiments in which the carbohydrate infusion rate was maintained more uniformly around 2.0 $\mathrm{Gm}$. per $\mathrm{Kg}$. per hour. Negative sodium balances were particularly prominent in the individual experiments in Group B in which the infusion rate varied above the mean for the group as a whole.

These indications of the existence of a metabolic acidosis were borne out by further investigation of blood $\mathrm{pH}, \mathrm{CO}_{2}$ and electrolyte changes. The probable mechanism for this acidosis was demonstrated to be an accumulation in the blood of lactic and pyruvic acids with increased urinary excretion of these metabolites. Similar elevation in blood levels of these intermediates has been reported previously from several sources $(8,17,18)$.

The present controlled studies show that the magnitude of these metabolic alterations is significantly greater with fructose administration than with glucose. The increased appearance of these metabolites occurring especially with fructose administration is probably a reflection of its rapid rate of utilization which has been attributed to a difference in rate of phosphorylation (17) and to the fact that fructose enters the Meyerhof pathway at a point further along than glucose (17). Smith, Ettinger, and Seligson (18) have suggested that the difference in acid metabolite accumulations is a reflection of the significant extent to which glucose oxidation in the human proceeds through the hexosemonophosphate shunt pathway, in which only $1 \mathrm{M}$ of pyruvate is formed from the breakdown of $1 \mathrm{M}$ of hexose, while $2 \mathrm{M}$ of pyruvate is produced in the Embden-Meyerhof pathway, which is the only known mechanism for fructose breakdown.

The subjects used in these studies had no preexisting acidosis, but showed blood $\mathrm{pH}$ decreases to as low as 7.11 during the course of a six hour infusion. Even more profound acidosis could be expected to occur in a patient who was acidotic before the infusion was begun. This situation might be encountered in a diabetic, acutely undernourished, or dehydrated infant.

The acidosis accompanying rapid fructose administration in infants has been shown to be of sufficient severity to make advisable limitation of its rate of infusion. No untoward side effects were 
noted with infusions of $1 \mathrm{Gm}$. per $\mathrm{Kg}$. per hour and no contraindication to its use at this rate has been demonstrated.

It should be pointed out that there may be indications for the use of fructose in special situations in which glucose utilization may be impaired: the initial hours of treatment of diabetic acidosis (20, 21), febrile illness (22), the early postoperative period (22), severe hepatic insufficiency (18), after fasting (23), and in the acidotic state (24). The present study does not yield information relevant to the validity of these indications.

\section{SUMMARY}

1. Infusions of glucose and fructose with electrolytes to three infants at a rate of approximately $1.0 \mathrm{Gm}$. per $\mathrm{Kg}$. per hour resulted in essentially complete utilization of both sugars. Excretion of water, sodium, and potassium did not vary significantly in relation to the sugar infused.

2. Infusions of the sugars at a rate of approximately $2.0 \mathrm{Gm}$. per $\mathrm{Kg}$. per hour in eight subjects resulted in a significantly greater urinary loss of sugar with glucose (20.0 per cent of intake) than with fructose $(9.9$ per cent) $(\mathrm{p}<0.02)$.

The average urinary sodium excretion associated with glucose infusion was 79 per cent of intake, which is significantly lower than that noted with fructose, 127 per cent $(p<0.01)$, but no significant differences in water and potassium excretions were noted.

Significant increases in pulse and respiratory rates and liver size occurred during fructose infusion in these subjects.

3. Infusions of fructose solution with electrolytes to seven additional infants at the rate of $2 \mathrm{Gm}$. per $\mathrm{Kg}$. per hour were accompanied by mean increases in whole blood lactate of $35.6 \mathrm{mg}$. per cent, and in pyruvate of $2.16 \mathrm{mg}$. per cent. Smaller increases occurred with glucose in eight subjects.

Decreases in blood $\mathrm{pH}$ up to 0.22 , and mean decreases in whole blood $\mathrm{CO}_{2}$ of $6.8 \mathrm{mM}$ per $\mathrm{L}$. and in plasma sodium of $14 \mathrm{mEq}$. per $\mathrm{L}$. were demonstrated in the group of individuals receiving fructose. Significantly smaller changes were seen in these blood levels during glucose infusion.

Mean lactic acid excretion during the period of infusion was $10.4 \mathrm{mg}$. per $\mathrm{Kg}$. per hour in the subjects receiving fructose, but only $0.6 \mathrm{mg}$. per $\mathrm{Kg}$. per hour in those receiving glucose.

4. It is suggested that the advantage in fructose utilization over glucose, when administered at a rapid rate of infusion, may be offset by the induced metabolic acidosis and its attendant side effects.

\section{ADDENDUM}

Since preparation of this manuscript, another study of the use of carbohydrates in parenteral feeding of children has been reported by Lane and Dodd (25). They administered 10 per cent solutions of glucose, invert sugar, and fructose, containing no electrolytes, to 18 children and found more glycosuria accompanying the glucose infusions than with the fructose or invert sugar in 16 of the subjects. Their rate of infusion was 1.25 $\mathrm{Gm}$. of sugar per $\mathrm{Kg}$. per hour, which is considerably lower than the $2 \mathrm{Gm}$. per $\mathrm{Kg}$. per hour rate which produced the significantly greater retention of fructose than glucose in the present study.

\section{ACKNOWLEDGMENTS}

The authors wish to express their appreciation to Drs. Seymour Cohen, Samuel Bessman, and David Seligson for their help in planning and interpreting these experiments.

\section{REFERENCES}

1. Weichselbaum, T. E., Elman, R., and Lund, R. H. Comparative utilization of fructose and glucose given intravenously. Proc. Soc. exp. Biol. (N. Y.) 1950, 75, 816.

2. Weinstein, J. J., and Roe, J. H. The utilization of fructose by human subjects and animals. J. Lab. clin. Med. 1952, 40, 39.

3. Bertino, J., Dawson, N., French, R., Margen, S., and Kinsell, L. W. Comparative observations regarding utilization and excretion of infused glucose, fructose, and invert sugar, respectively. J. clin. Endocr. 1953, 13, 658.

4. Strub, I. H., Best, W. R., Consolazio, C. F., and Grossman, M. I. Utilization of intravenously injected fructose and invert sugar in normal human subjects. Amer. J. clin. Nutr. 1954, 2, 32.

5. Jonxis, J. H. P., and Huisman, T. H. J. The excretion of sugars after the intravenous administration of invert sugar. Arch. Dis. Childh. 1953, 28, 446.

6. Beal, J. M., Smith, J. L., and Frost, P. M. Studies in the utilization of fructose administered intravenously in man. Surgery 1953, 33, 721. 
7. Butler, A. M., and Talbot, N. B. Parenteral fluid therapy. I. Estimation and provision of daily maintenance requirements. New Engl. J. Med. 1944, 231, 585.

8. Weichselbaum, T. E., Margraf, H. W., and Elman, R. Metabolism of intravenously infused fructose in man. Metabolism 1953, 2, 434.

9. Kruesi, O. R., Goodbody, M. F., Jr., Van Itallie, T. B., and Hilton, J. G. Effect of intravenously administered fructose on blood acid-base balance in patients with pre-existing acidosis. Diabetes 1955, 4, 104.

10. Nelson, N. A photometric adaptation of the Somogyi method for the determination of glucose. J. biol. Chem. 1944, 153, 375.

11. Schreiner, G. E. Determination of inulin by means of resorcinol. Proc. Soc. exp. Biol. (N. Y.) 1950, 74, 117.

12. Peters, J. P., and Van Slyke, D. D. Quantitative Clinical Chemistry. Baltimore, Williams and Wilkins, 1932, vol. II.

13. Schales, O., and Schales, S. S. A simple and accurate method for the determination of chloride in biological fluids. J. biol. Chem. 1941, 140, 879.

14. Barker, S. B., and Summerson, W. H. The colorimetric determination of lactic acid in biological material. J. biol. Chem. 1941, 138, 535.

15. Friedemann, T. E., and Haugen, G. E. Pyruvic acid II. The determination of keto acids in blood and urine. J. biol. Chem. 1943, 147, 415.

16. Higashi, A., and Peters, L. A rapid colorimetric method for the determination of inulin in plasma and urine. J. Lab. clin. Med. 1950, 35, 475.

17. Miller, M., Drucker, W. R., Owens, J. E., Craig, J. W., and Woodward, H., Jr. Metabolism of intravenous fructose and glucose in normal and diabetic subjects. J. clin. Invest. 1952, 31, 115.

18. Smith, L. H., Jr., Ettinger, R. H., and Seligson, D. A comparison of the metabolism of fructose and glucose in hepatic disease and diabetes mellitus. J. clin. Invest. 1953, 32, 273.

19. Cori, C. F. The fate of sugar in the animal body. III. The rate of glycogen formation in the liver of normal and insulinized rats during the absorption of glucose, fructose, and galactose. J. biol. Chem. 1926, 70, 577.

20. Darragh, J. H., Womersley, R. A., and Meroney, W. H. Fructose in the treatment of diabetic ketosis. J. clin. Invest. 1953, 32, 1214.

21. Miller, M., Murphy, J. R., Craig, J. W., and Woodward, H., Jr. Studies in experimental diabetic acidosis: Comparison of the effect of fructose and glucose in the initial hours of treatment. J. clin. Endocr. 1953, 13, 866.

22. Drucker, W. R., Miller, M., Abbott, W. E., Craig, J. W., Jefferies, Wm. McK., Levey, S., and Woodward, H., Jr. The effect of stress on glucose and fructose metabolism. J. Lab. clin. Med. 1952, 40, 794.

23. Miller, M., Craig, J. W., and Woodward, H., Jr. The effect of carbohydrate deprivation on the metabolism of fructose and glucose in normal human subjects. J. clin. Invest. 1953, 32, 591.

24. Mackler, B., and Guest, G. M. Effects of acidosis on the metabolism of fructose. Amer. J. Physiol. 1953, 174, 54.

25. Lane, H. C., and Dodd, K. Use of glucose, invert sugar and fructose for parenteral feeding of children. Pediatrics 1957, 20, 668. 